\title{
A study on trajectory control of manipulator using power packet dispatching
}

\section{$\operatorname{AUTHOR}(\mathrm{S})$ :}

Mochiyama, Shiu; Fujii, Naoaki; Takahashi, Ryo; Hikihara, Takashi

\section{CITATION:}

Mochiyama, Shiu ...[et al]. A study on trajectory control of manipulator using power packet dispatching. Telecommunications Energy Conference (INTELEC), 2015 IEEE International 2016: 7572460.

ISSUE DATE:

2016-09-20

URL:

http://hdl.handle.net/2433/237406

\section{RIGHT:}

(c) 2015 IEEE. Personal use of this material is permitted. Permission from IEEE must be obtained for all other uses, in any current or future media, including reprinting/republishing this material for advertising or promotional purposes, creating new collective works, for resale or redistribution to servers or lists, or reuse of any copyrighted component of this work in other works.; This is not the published version. Please cite only the published version.; この論文は出版社版

でありません。引用の際には出版社版をご確認ご利用ください。 


\title{
A Study on Trajectory Control of Manipulator Using Power Packet Dispatching
}

\author{
Shiu Mochiyama Naoaki Fujii Ryo Takahashi Takashi Hikihara \\ Department of Electrical Engineering, Kyoto University, Kyoto-shi, 615-8510 Japan \\ E-mail: s-mochiyama@dove.kuee.kyoto-u.ac.jp
}

\begin{abstract}
In the power packet dispatching system, electric power is transferred with information tags attached physically. An application of the system to robots can enable flexible and efficient power management with a dynamically varying power-line connection between power sources and loads. In this paper, a numerical simulation verifies the achievement of a trajectory control of a manipulator by power packets. As a result, it is clarified that a conventional power conversion system at the load side can be replaced by a packet generating system at the source side. This can be a step for new power management inside robots with power packets.
\end{abstract}

\section{Introduction}

Today robots are utilized in a variety of areas. Many of these robots use electric power as a power supply. They usually have multiple loads, such as electric motors, and a power source. These robots generally have a fixed power-line connection between a power source and loads. The static connection will be a target of improvement concerning power management inside robots. It includes an effective use of distributed power sources and power management with limited power available. The power system inside robots needs to be reconsidered for satisfying the requests.

As a way of energy management with information technologies, several studies have discussed a routing of electric power [1-9]. Among them, in 1990s, Toyoda and Saitoh proposed an open electric energy network (OEEN) as a method to manage electric power flow on utility grids in the same way as a postal packet delivery with an information tag [1]. Unfortunately this proposal was too early because the technology of power electronics was not matured at that time. Recent development of wide band gap semiconductor devices $[10,11]$ encouraged us to extend the concept and propose a power packet dispatching system [4-9]. In the power packet dispatching system, pulse power is transferred with information tags attached physically and using a time division multi-plexing (TDM) method. The power packet are delivered through power routers by putting information, such as a destination address, to a tag of a packet. In $[8,9]$, power packet dispatching on a networked system has been verified experimen-

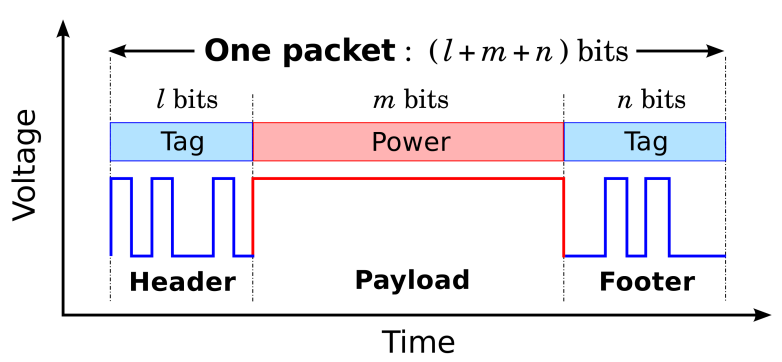

Figure 1: Configuration of power packet.

tally. The system has a possibility to realize a power transfer with flexible power-line connections between sources and loads. It may change the method for power delivery from the way of continuous flow as water to the quantized parcels as bottles.

Recently, the possibility of power packets to drive and control a robot has been discussed. For example, an algorithm for power packet generation to meet demands of loads in the dispatching system has been developed [12]. In addition, it has been demonstrated in experiments that a stepper motor can be driven by transferred power packets [13].

In this paper, we discuss a manipulator control with power packets. The manipulator consists of multiple motors driven by electric power. Power packets are supplied to the motors so that the trajectory of the manipulator tip can track a desired trajectory. The achievement of the manipulator control and the possibility of the power management are confirmed in a numerical simulation.

\section{Power Packet Dispatching System}

Figure 1 shows the configuration of a power packet. A power packet consists of a payload and information tags. A payload is a dc power pulse, and the amount of transferred power can be arbitrarily decided by changing the length or modulating the pulse itself. An information tag, composed of a header and a footer, is attached to the payload in voltage waveforms. The information transferred by tags can include an identifier of the source of the packet, the destination address and so on. The tags can be in arbitrary length. 


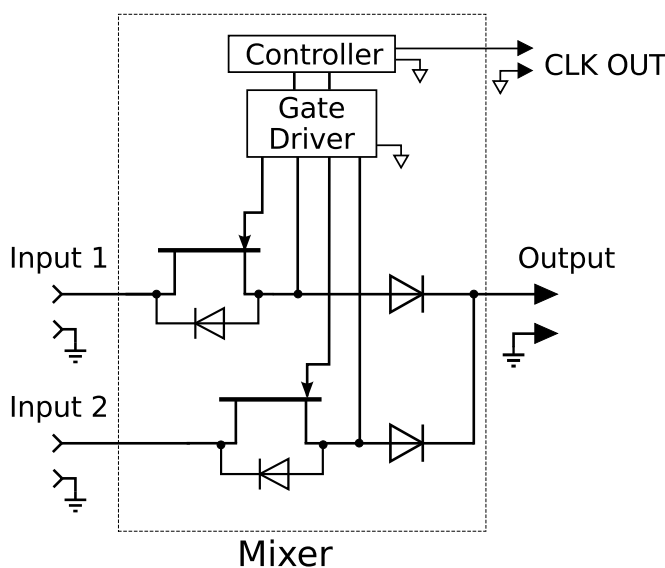

Figure 2: Configuration of power mixer.

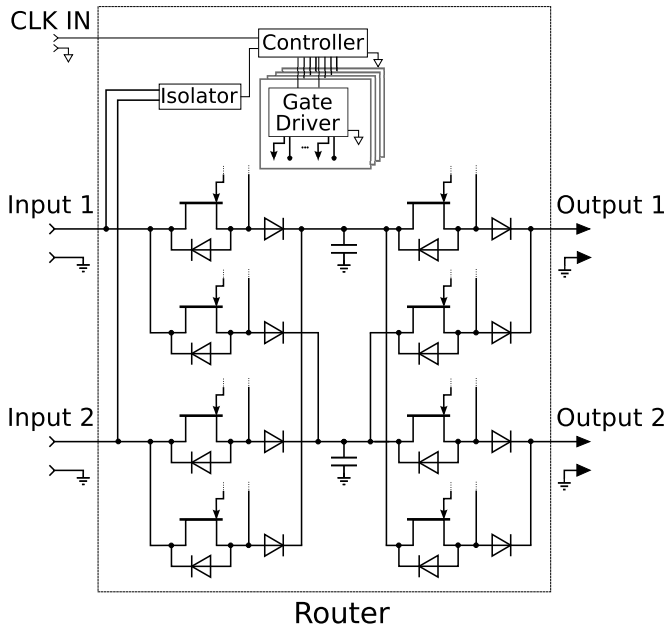

Figure 3: Configuration of power router.

In [7-9], power packet dispatching network was designed and verified experimentally. The network contains power packet mixers and routers. Figures 2 and 3 depict configurations of a mixer and a router, respectively. The mixers produce power packets by a circuit switching. Note that SiC JFETs enables a power switching at several $\mathrm{MHz}$ [11]. Thus the length of information bits can be sufficiently shorter than that of a payload. Then the produced packets are delivered with TDM method; that is, mixers do not send two or more packets on a single power-line simultaneously. In this way, packets from different sources will not mix each other on the line. When a router receives a packet, it reads the information in the header, and dispatches the packet to the desired destination according to the information.

Here, the capacitors in the router work as buffers for reproduction of information tags $[8,9]$. Buffers can be prepared for each power source, and the switches in the input side guides input packets to an appropriate buffer. When all bits of the header are read, packets with a reproduced header is made by the switch in the output side connected to the desired destination. The system achieves "stored and forward."

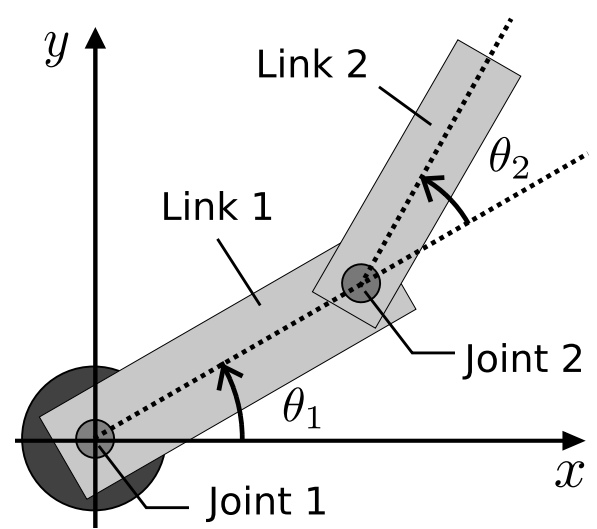

Figure 4: Configuration of 2 DOF manipulator.

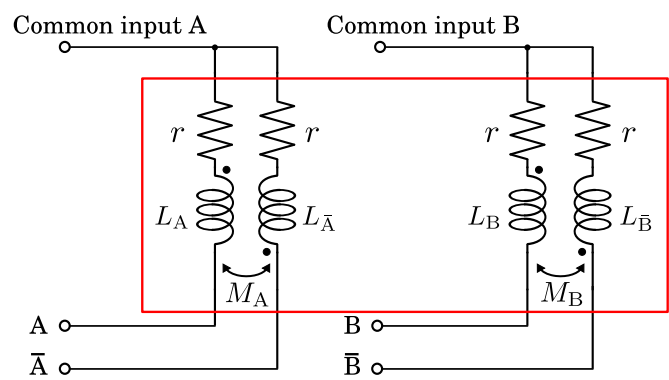

Figure 5: Electrical circuit diagram of a stepper motor.

\section{Trajectory Control of Manip- ulator}

\subsection{Mathematical Models of Manipula- tor}

Figure 4 shows a two degrees of freedom manipulator. The manipulator tip moves in a plane as two electric motors at each joints rotate independently. The motor at joint 1 is fixed on a horizontal base plane. Dynamic equations in terms of joint displacements $\theta_{1}$ and $\theta_{2}$, and joint torques $\tau_{1}$ and $\tau_{2}$ are given as follows [14]:

$$
\begin{aligned}
& \tau_{1}=H_{11} \ddot{\theta}_{1}+H_{12} \ddot{\theta}_{2}+h_{122} \dot{\theta}_{2}^{2}+h_{112} \dot{\theta}_{1} \dot{\theta}_{2}+c_{1} \dot{\theta}_{1}, \\
& \tau_{2}=H_{21} \ddot{\theta}_{1}+H_{22} \ddot{\theta}_{2}+h_{211} \dot{\theta}_{1}{ }^{2}+c_{2} \dot{\theta}_{2},
\end{aligned}
$$

where coefficients $H$ and $h$ are functions of $\theta_{2}$, and $c$ is a friction constant.

The manipulator contains unipolar hybrid stepper motors at its joints. We call the motor at joint 1 and at joint 2 as motor 1 and motor 2 , respectively. The motors have different rating currents: $3.3 \mathrm{~A}$ for motor 1 and $0.95 \mathrm{~A}$ for motor 2 . The motors can be expressed as the electrical circuit shown in Fig. 5. The circuit equation for each phase in terms of input voltage $V$ 


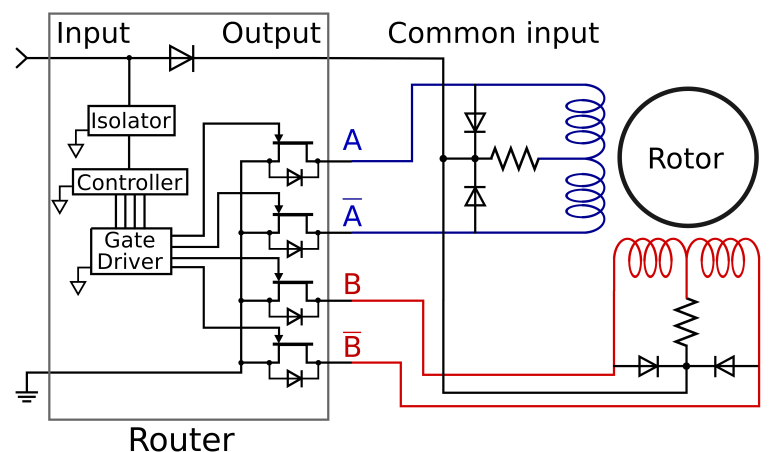

Figure 6: Configuration of routing circuit for stepper motor drive proposed in [13].

and current $i$ is given as follows:

$$
\begin{aligned}
& V_{\mathrm{A}}-r i_{\mathrm{A}}-L_{\mathrm{A}} \frac{\mathrm{d} i_{\mathrm{A}}}{\mathrm{d} t}-M_{\mathrm{A}} \frac{\mathrm{d} i_{\overline{\mathrm{A}}}}{\mathrm{d} t}+e_{\mathrm{MA}}=0, \\
& V_{\overline{\mathrm{A}}}-r i_{\overline{\mathrm{A}}}-L_{\overline{\mathrm{A}}} \frac{\mathrm{d} i_{\overline{\mathrm{A}}}}{\mathrm{d} t}-M_{\mathrm{A}} \frac{\mathrm{d} i_{\mathrm{A}}}{\mathrm{d} t}+e_{\mathrm{MA}}=0, \\
& V_{\mathrm{B}}-r i_{\mathrm{B}}-L_{\mathrm{B}} \frac{\mathrm{d} i_{\mathrm{B}}}{\mathrm{d} t}-M_{\mathrm{B}} \frac{\mathrm{d} i_{\overline{\mathrm{B}}}}{\mathrm{d} t}+e_{\mathrm{MB}}=0, \\
& V_{\overline{\mathrm{B}}}-r i_{\overline{\mathrm{B}}}-L_{\overline{\mathrm{B}}} \frac{\mathrm{d} i_{\overline{\mathrm{B}}}}{\mathrm{d} t}-M_{\mathrm{B}} \frac{\mathrm{d} i_{\mathrm{B}}}{\mathrm{d} t}-e_{\mathrm{M} \overline{\mathrm{B}}}=0,
\end{aligned}
$$

where $r, L$ and $M$ denote a resistance, an inductance and a mutual inductance of a motor winding, respectively.

The stepper motors rotate according to the input current sequence, and the rotational torque is proportional to an amplitude of the motor currents. The torque is given as follows:

$\tau=-p K_{\mathrm{e}}\left(i_{\mathrm{A}}-i_{\overline{\mathrm{A}}}\right) \sin p \theta-p K_{\mathrm{e}}\left(i_{\mathrm{B}}-i_{\overline{\mathrm{B}}}\right) \sin p(\theta-\lambda)$,

where $p, \lambda$ and $K_{\mathrm{e}}$ denote constants determined by the motor specification.

The motors are driven with power packets using a routing circuit proposed in [13]. Figure 6 depicts the schematic of the routing circuit. The router contains four switches, each of which corresponds to every single excitation phase. The router reads the phaseexcitation information in the header of input packets and turn on the switch(es) for the desired excitation phase(s).

\subsection{Simulation Setting}

Figure 7 shows the schematic of the simulation circuit. The mixer produces a sequence of power packets so that the motors can track given trajectories. Each packet has a destination address in its header and is transferred to one of the motors. Note that the Router $\mathrm{C}$ has a buffer and can reproduce tags so that an arbitrary router network can be connected to the output of Router C. In this paper, we examine the simplest case, where the network contains one router and one load.

The length of each power packet is $20 \mu \mathrm{s}$, and $2 \mu \mathrm{s}$ of them are used for the information tags. We assume electric power is not supplied during the tags at all.

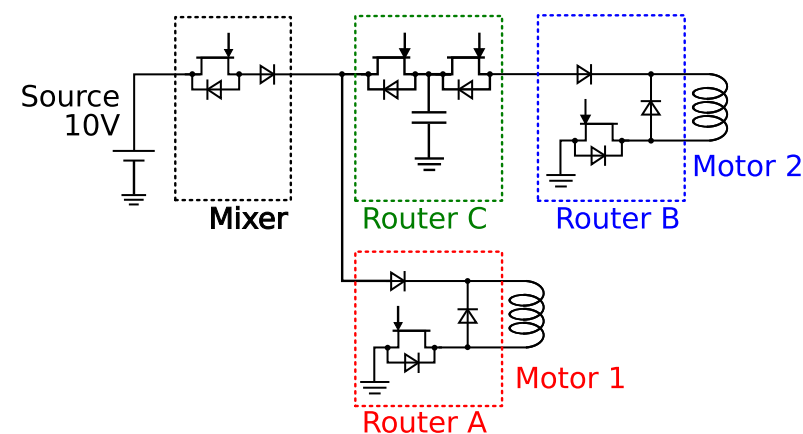

Figure 7: Configuration of simulation circuit.

The packet length is sufficiently shorter than the phase transition period of the desired current sequence. Thus the power supplied to the motor can be regulated by modulating the density of power packets with an application of an optimal dynamic quantizer (ODQ) proposed in $[12,15]$. The necessity of a power packet for each motor is determined by the dynamic quantizer in the mixer every $20 \mu \mathrm{s}$. From (3)-(6), the plant $P$, or the discretized electric circuit equations are given as follows:

$$
P:\left\{\begin{array}{l}
\boldsymbol{x}(k+1)=\boldsymbol{A} \boldsymbol{x}(k)+\boldsymbol{B} \boldsymbol{v}(k), \\
\boldsymbol{y}(k)=\boldsymbol{C} \boldsymbol{x}(k)
\end{array},\right.
$$

where $\boldsymbol{A}, \boldsymbol{B}, \boldsymbol{C} \in \mathbb{R}^{4 \times 4}$ are constant matrices, and vectors $\boldsymbol{x} \in \mathbb{R}^{4}, \boldsymbol{v} \in \mathbb{R}^{4}$ and $\boldsymbol{y} \in \mathbb{R}^{4}$ represent the state, control input and output respectively. The input and output have a unit of voltage. The three vectors contain a value for phase $\mathrm{A}, \mathrm{B}, \overline{\mathrm{A}}$ and $\overline{\mathrm{B}}$ in their first, second, third and fourth element respectively. Then the dynamic quantizer $Q$ for the plant $P$ is obtained as follows:

$$
Q:\left\{\begin{array}{l}
\boldsymbol{\xi}(k+1)=\boldsymbol{A} \boldsymbol{\xi}(k)-\boldsymbol{B} \boldsymbol{u}(k)+\boldsymbol{B} \boldsymbol{v}(k) \\
\boldsymbol{v}(k)=q\left(-(\boldsymbol{C B})^{-1} \boldsymbol{C} \boldsymbol{A} \boldsymbol{\xi}(k)+\boldsymbol{u}(k)\right)
\end{array},\right.
$$

where $q$ is a static quantizer of quantization interval $d$, and $\boldsymbol{\xi} \in \mathbb{R}^{4}, \boldsymbol{u} \in \mathbb{R}^{4}$ and $\boldsymbol{v} \in d \mathbb{Z}^{4}$ represent the state, input and output respectively. The output corresponds to the input of $P$, and the input of $Q$ to the desired current sequence.

Power packets are transferred with TDM, and every single packet can be supplied to one destination load. Thus a selector is also introduced into the packet generation algorithm in the mixer with the ODQ. The selector determines which motor a packet should be supplied to when both motors demand the packet. For this purpose, we focus on the vector $C A \xi(k)$, which expresses the effect of quantization errors on the output of $P$. The selector compares the value of $\|C A \xi(k)\|_{1}$, the 1-norm of $C A \xi(k)$, of each motor. The packet is supplied to the motor whose value is greater than the other.

With the preparations above, we conducted a numerical simulation using MATLAB. The simulation includes solving three sets of differential equations as shown in Fig. 8. The desired sequence is determined according to the desired trajectory and is applied to 


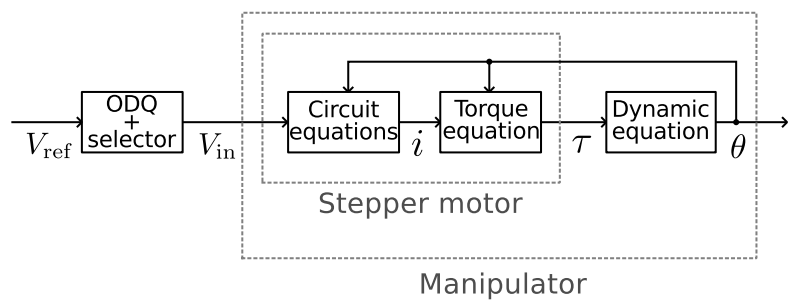

Figure 8: Outline of simulation flow.

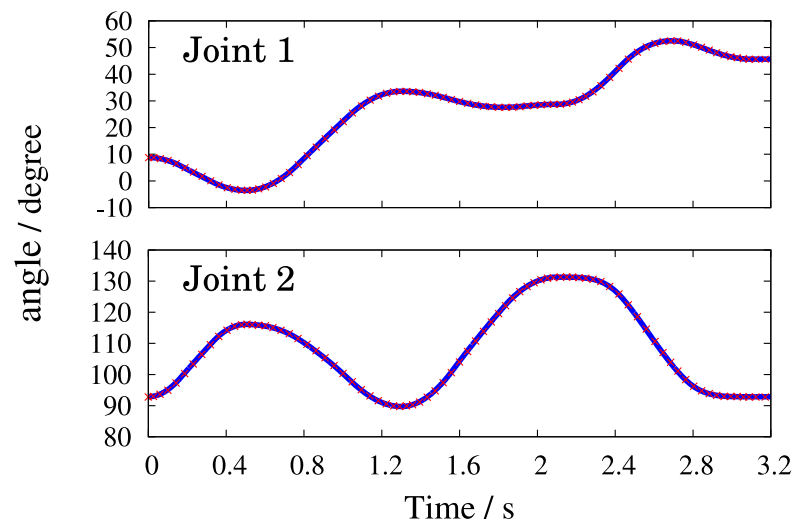

Figure 9: Rotation angles (red plots) and their reference angle trajectories (blue lines).

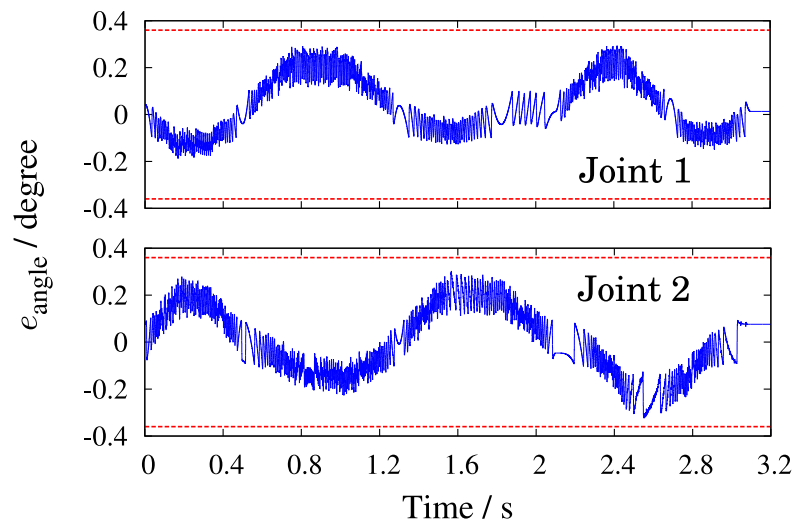

Figure 10: Angle errors (blue lines) and their allowable limits (red lines).

ODQ. The ODQ determines the input of electric circuit. Then, using (3)-(6), the circuit equation is solved to obtain the motor current. The motor current determines the motor torque through (7). The torque is an input to the dynamic equations (1), (2). The output of the dynamic equation is not only an output of the whole system but an input of circuit equation (3) and torque equation (7).

\subsection{Results of Simulation}

First, the trajectory of the manipulator is examined. Figure 9 shows the rotation angle of each joint and its reference angle trajectory. The angle (red plots) successfully tracked the desired trajectory (blue line) throughout the simulation. To examine the details of angle trajectory, we check $e_{\text {angle }}$, the gap between joint angles and their reference trajectories shown in Fig. 10.
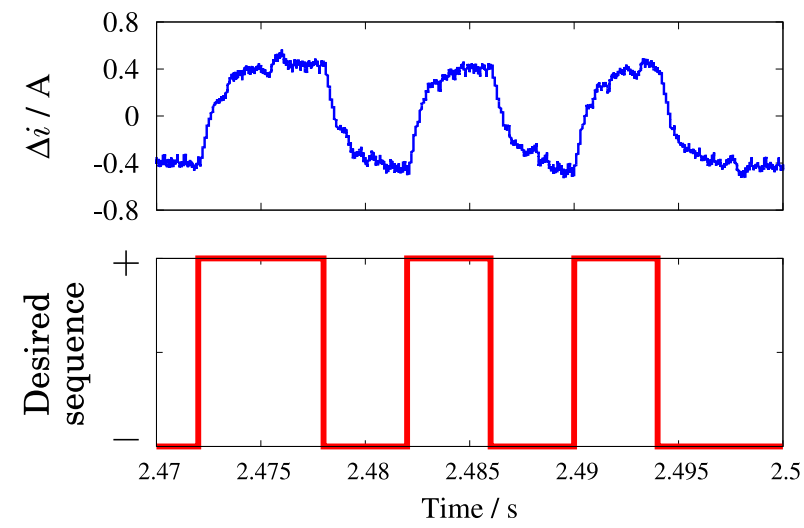

Figure 11: Current waveform and its desired sequence.

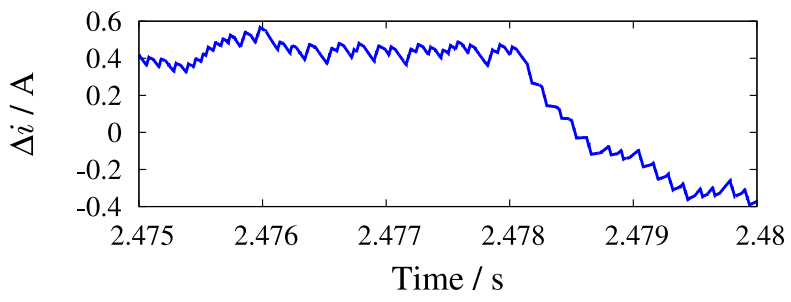

Figure 12: Enlarged view of current waveform and its reference sequence.

The blue line represents $e_{\text {angle, }}$, and red lines the upper and lower limits of possible angle error. The limits are determined prior to the simulation by considering the change rate of reference trajectory and mechanical characteristics of the motors. In this simulation we determined the limit as $\pm 0.36^{\circ}$. Figure 10 shows that the gaps stayed inside the limits throughout the simulation. Therefore, the achievement of trajectory control is confirmed.

Second, the motor current waveforms are examined. As shown in (7), the motor torque is determined by the difference of phase currents such as $\Delta i_{\mathrm{A}}:=i_{\mathrm{A}}-i_{\overline{\mathrm{A}}}$. Figure 11 shows $\Delta i_{\mathrm{A}}$ of motor 2 and its reference current sequence. Although the current rise and fall took some transient time because of the inductive loads, the waveform followed the desired phase-excitation sequence.

Figure 12 is the enlarged view of Fig. 11 from $2.475 \mathrm{~s}$ to $2.48 \mathrm{~s}$. The current repeated to rise and fall in the shorter period than the desired sequence changed. This indicates that power packets were generated and supplied in appropriate intervals so that the current were regulated to be the desired value.

\section{Conclusions}

In this paper, it has been numerically verified that the trajectory control is achieved with a regulation of power supplied by power packets. The ODQ generated power packets with consideration of the different power demands of each motor. The selector guaranteed that individual packets were transferred to the desired motor without mixing each other. These results clarify that we can replace a conventional power conversion 
at the load with a packet generation at power sources. These discussions open the way to adopt the power supply system under optimizations for a robot and related independent systems.

\section{Acknowledgement}

This work was partially supported by Cross-ministerial Strategic Innovation Promotion Program from New Energy and Industrial Technology Development Organization and by the Super Cluster Program from Japan Science and Technology Agency.

\section{References}

[1] J. Toyoda and H. Saitoh, "Proposal of an openelectric-energy-network (OEEN) to realize cooperative operations of IOU and IPP," in Proc. 1998 Int. Conf. Energy Manage. Power Del. (EMPD), vol. 1, Singapore, 1998, pp. 218-222.

[2] M. M. He et al., "An architecture for local energy generation, distribution, and sharing," in Proc. IEEE Energy 2030 Conf., Atlanta, GA, USA, Nov 2008, pp. 1-6.

[3] E. Gelenbe, "Energy packet networks: Adaptive energy management for the cloud," in Proc. 2nd Int. Workshop Cloud Comput. Platforms, Bern, Switzerland, 2012.

[4] T. Hikihara, "Power router and packetization project for home electric energy management," Apr. 2010, presented at the Santa Barbara Summit Energy Efficiency, Santa Barbara, CA, USA, April, 2010.

[5] T. Hikihara, K. Tashiro, Y. Kitamori, and R. Takahashi, "Power packetization and routing for smart management of electricity," in Proc. AIAA 10th Int. Energy Convers. Eng. Conf. (IECEC), Atlanta, GA, USA, 2012, pp. 1-6.

[6] T. Takuno, M. Koyama, and T. Hikihara, "In-home power distribution systems by circuit switching and power packet dispatching," in Proc. 1st IEEE Int. Conf. Smart Grid Commun., Gaithersburg, MD, USA, Oct. 2010, pp. 427-430.

[7] K. Tashiro, R. Takahashi, and T. Hikihara, "Feasibility of power packet dispatching at in-home DC distribution network," in Proc. 3rd IEEE Int. Conf. Smart Grid Commun., Tainan, Taiwan, Nov. 2012, pp. 401-405.

[8] N. Fujii, R. Takahashi, and T. Hikihara, "Networked power packet dispatching system for multipath routing," in Proc. IEEE/SICE Int. Symp. System Integr., Tokyo, Japan, Dec. 2014, pp. 357362.
[9] R. Takahashi, K. Tashiro, and T. Hikihara, "Router for power packet distribution network: Design and experimental verification," IEEE Trans. Smart Grid, vol. 6, no. 2, pp. 618-626, Mar. 2015.

[10] T. Funaki et al., "Power conversion with SiC devices at extremely high ambient temperatures," IEEE Trans. Power Electron., vol. 22, no. 4, pp. 1321-1329, Jul. 2007.

[11] T. Takuno, T. Hikihara, T. Tsuno, and S. Hatsukawa, "HF gate drive circuit for a normallyon SiC JFET with inherent safety," in Proc. 13th Eur. Conf. Power Electron. Appl., Barcelona, Spain, Sep. 2009, pp. 1-4.

[12] R. Takahashi, S.-i. Azuma, K. Tashiro, and T. Hikihara, "Design and experimental verification of power packet generation system for power packet dispatching system," in Proc. Amer. Control Conf., Washington, DC, Jun. 2013, pp. 43684373.

[13] N. Fujii, R. Takahashi, and T. Hikihara, "Application of power packet dispatching system to stepping motor driving system," in The Papers of Joint Tech. Meeting on Electron. Devices and Semicond. Power Converter, IEE Jpn., Oct. 2014, pp. 53-58, in Japanese.

[14] H. Asada and J.-J. E. Slotine, Robot Analysis and Control, 1st ed. USA: John Wiley \& Sons, 1986.

[15] S.-i. Azuma and T. Sugie, "Optimal dynamic quantizers for discrete-valued input control," $A u$ tomatica, vol. 44, no. 2, pp. 396-406, Feb. 2008. 